\title{
Coherence correlations in the dissipative two-state system
}

\author{
Gunther Lang ${ }^{1}$, Elisabetta Paladino ${ }^{2,1}$, and Ulrich Weiss ${ }^{1}$ \\ 1 Institut für Theoretische Physik, Universität Stuttgart, 70550 Stuttgart, Germany \\ ${ }^{2}$ Istituto di Fisica, Università di Catania \& INFM, Viale A. Doria 6, 95129 Catania, Italy.
}

\begin{abstract}
We study the dynamical equilibrium correlation function of the polarondressed tunneling operator in the dissipative two-state system. Unlike the position operator, this coherence operator acts in the full system-plus-reservoir space. We calculate the relevant modified influence functional and present the exact formal expression for the coherence correlations in the form of a series in the number of tunneling events. For an Ohmic spectral density with the particular damping strength $K=\frac{1}{2}$, the series is summed in analytic form for all times and for arbitrary values of temperature and bias. Using a diagrammatic approach, we find the long-time dynamics in the regime $K<1$. In general, the coherence correlations decay algebraically as $t^{-2 K}$ at $T=0$. This implies that the linear static susceptibility diverges for $K \leq \frac{1}{2}$ as $T \rightarrow 0$, whereas it stays finite for $K>\frac{1}{2}$ in this limit. The qualitative differences with respect to the asymptotic behavior of the position correlations are explained.
\end{abstract}

\section{INTRODUCTION}

The simplest model that allows to study the interplay of tunneling and dissipation is the spin-boson model [1,2]. Despite its simplicity, it exhibits generic features of many complex systems and has found widespread applications in physics and chemistry. It has been adopted to describe diverse systems, like the tunneling of atoms between a surface and the tip of an atomic-force microscope [3], or the dynamics of the trapped flux in a quantum interference device [4], to mention a few.

For Ohmic dissipation, the spin-boson model shows a transition between coherent and incoherent tunneling where the location of the transition depends on the damping strength and on the bias [2]. Most investigations have been done for the non-equilibrium expectation value $\left\langle\sigma_{z}(t)\right\rangle$, representing the population difference between the two localized states, and for the $\sigma_{z}$ autocorrelation function, describing position or population correlations [5,6]. For the latter quantity, the analogy with the Kondo model and the $1 / r^{2}$ Ising model has been utilized [7] 9] in numerical computations. For Ohmic damping and zero temperature and bias, $\left\langle\sigma_{z}(t)\right\rangle$ shows a transition between damped oscillations and incoherent relaxation exactly at $K=\frac{1}{2}$ [10]. Recently, it has been argued [11] that the quality factor of the oscillation is exactly given by $Q=\cot [\pi K / 2(1-K)]$, which again gives $K=\frac{1}{2}$ for the transition. For the antisymmetrized $\sigma_{z}$ autocorrelation function, the same quality factor and thus the same transition point was found numerically [9]. This is not in contrast to the earlier result $K=\frac{1}{3}[8,12]$, since there a different criterion for the transition was applied [9,13].

It has been shown that the particular initial preparation plays a crucial role for the long-time behavior at zero temperature. The factorized system-reservoir initial state for the 
expectation value $\left\langle\sigma_{z}(t)\right\rangle$ leads to exponential decay [10,11], whereas the symmetrized $\sigma_{z}$ equilibrium correlation function with a correlated initial state decays algebraically as $1 / t^{2}$ for $K<1$, as shown for the spin-boson model [6] and the related $1 / r^{2}$ Ising and fermionic models $[7,8,12]$. The power 2 in the algebraic decay law is a signature of Ohmic dissipation.

Recently, focus has been put on expectation values [14] and equilibrium correlations [15] connected with the tunneling operator $\sigma_{x}$. The expectation value $\left\langle\sigma_{x}(t)\right\rangle$ and the equilibrium autocorrelation function of $\sigma_{x}$ have been found to be nonuniversal, i.e., vanish in the scaling limit 14,16]. Here, we study the equilibrium autocorrelation function of a polaron-dressed tunneling operator $\tilde{\sigma}_{x}$ which includes the adiabatic dynamics of the bath modes [1, 17]. This function is universal and measures correlations of the off-diagonal elements (coherences) of the density matrix. We present the exact formal solution for the coherence correlations in the form of a series in the number of tunneling events. We then analyze the resulting expression in various limits. In particular, we work out the differences in the asymptotic decay between the position correlations and the coherence correlations. Our analytical real-time approach is complementary to the recent imaginary-time numerical studies in Ref. [15].

In Section II, we formulate the problem and introduce the correlation function $C_{x}(t)$ of the coherence operator $\tilde{\sigma}_{x}$. Since this operator acts both in the system and bath space, the elimination of the bath modes has to be reconsidered. The relevant considerations leading to a modified influence functional are given in Section III. These results are used in Section IV to determine exact formal expressions for $C_{x}(t)$. In Section $\mathrm{V}$, we present the analytical solution for $C_{x}(t)$ for the special value $K=\frac{1}{2}$. Section VI is devoted to the regime $K=\frac{1}{2}-\kappa$ with $\kappa \ll 1$. Finally, in Section VII we show that the asymptotic decay of $C_{x}(t)$ at zero temperature is algebraic with a $K$-dependent power for $0<K<1$.

\section{FORMULATION OF THE PROBLEM}

It has been well established that the dissipative dynamics of a particle in a double-well potential can effectively be described at very low $T$ by the spin-boson model [1,2]

$$
\begin{aligned}
H & =H_{0}+\sum_{\alpha}\left[\frac{p_{\alpha}^{2}}{2 m_{\alpha}}+\frac{1}{2} m_{\alpha} \omega_{\alpha}^{2}\left(x_{\alpha}-\frac{c_{\alpha}}{m_{\alpha} \omega_{\alpha}^{2}} \frac{a}{2} \sigma_{z}\right)^{2}\right], \\
H_{0} & =-\hbar\left(\Delta \sigma_{x}+\epsilon \sigma_{z}\right) / 2 .
\end{aligned}
$$

Here the basis is formed by the two localized states $|R\rangle$ and $|L\rangle$ of the double-well which are eigenstates of $\sigma_{z}$ with eigenvalues +1 and -1 , respectively. The position operator is $q=a \sigma_{z} / 2$ with $\sigma_{z}=|R\rangle\langle R|-| L\rangle\langle L|$. The tunneling operator $\sigma_{x}=|R\rangle\langle L|+| L\rangle\langle R|$ transfers the particle between the two wells with tunneling amplitude $\Delta$. The second term in Eq. (2.2) describes an externally applied bias energy $\epsilon$. The effect of the thermal bath on the system's dynamics is included in the spectral density

$$
J(\omega)=\frac{\pi}{2} \sum_{\alpha} \frac{c_{\alpha}^{2}}{m_{\alpha} \omega_{\alpha}} \delta\left(\omega-\omega_{\alpha}\right) .
$$

The important case of an Ohmic bath is described by

$$
J(\omega)=\eta \omega e^{-\omega / \omega_{c}}=\left(2 \pi \hbar K / a^{2}\right) \omega e^{-\omega / \omega_{c}},
$$


where $\eta$ is the viscosity, $K$ is the appropriate dimensionless damping strength, and $\omega_{c}$ is a cut-off for the bath modes. We are interested in the regime $\Delta \ll \omega_{c}$, in which $\Delta$ and $\omega_{c}$ form a renormalized frequency scale [1]

$$
\Delta_{r}=\Delta\left(\Delta / \omega_{c}\right)^{K /(1-K)}
$$

A quantity is called universal, if it is a function of $\Delta_{r}$ alone, i.e., there is no other $\omega_{c}$ dependence than given by Eq. (2.5). Vice-versa, any extra dependence on $\omega_{c}$ is non-universal: performing the scaling limit $\Delta_{r} / \omega_{c} \rightarrow 0$ with $\Delta_{r}$ fixed, this contribution vanishes. Both the mean value of $\sigma_{z}$ and its equilibrium autocorrelation function are universal [1, 5]. On the other hand, the expectation value $\left\langle\sigma_{x}(t)\right\rangle$ is equipped with an overall factor $\Delta_{r} / \Delta=$ $\left(\Delta_{r} / \omega_{c}\right)^{K}$ and therefore vanishes in the scaling limit [14.

Here we concentrate on the $\sigma_{x}$ equilibrium autocorrelation function. As observed in Ref. [16] and explained in the sequel, the equilibrium correlation function of the bare $\sigma_{x}$ does not satisfy the above universality criterion. To overcome this shortcoming, we consider a modified tunneling operator which takes into account the adiabatic displacement of the bath modes during the tunneling process. The transformation to a basis of displaced harmonic oscillators states is accomplished by the polaron unitary transformation [1]

$$
\begin{aligned}
& U=\exp \left\{-i \sigma_{z} \Omega / 2 \hbar\right\} \\
& \Omega=a \sum_{\alpha} \frac{c_{\alpha}}{m_{\alpha} \omega_{\alpha}^{2}} p_{\alpha}=\sum_{\alpha} s_{\alpha} p_{\alpha} .
\end{aligned}
$$

The set of displacements is given by $\left\{s_{\alpha}\right\} \equiv\left\{a c_{\alpha} / m_{\alpha} \omega_{\alpha}^{2}\right\}$. The polaron transformed tunneling operator $\tilde{\sigma}_{x}=U \sigma_{x} U^{-1}$ reads

$$
\begin{aligned}
\tilde{\sigma}_{x} & =|R\rangle\langle L|\exp \{-i \Omega / \hbar\}+| L\rangle\langle R| \exp \{i \Omega / \hbar\} \\
& =|R\rangle\left\langle L\left|\int d x\right| x\right\rangle\langle x-s|+\text { h. c. },
\end{aligned}
$$

where we have introduced the compact notation

$$
s \equiv\left\{s_{\alpha}\right\}, \quad x \equiv\left\{x_{\alpha}\right\}, \quad \int d x \equiv \prod_{\alpha} \int d x_{\alpha} .
$$

The bare $\sigma_{x}$ acts in the Hilbert space of the two-state system (TSS) alone, whereas the dressed operator $\tilde{\sigma}_{x}$ acts in the full system-plus-reservoir space. From the coordinate representation (2.8), we immediately see that the operation of $\tilde{\sigma}_{x}$ transfers the particle from one localized state to the other and simultaneously shifts each bath-oscillator by the displacement $\pm s_{\alpha}$ ("polaronic cloud"). In this sense, $\tilde{\sigma}_{x}$ generates coherent tunneling between the two localized states and can be called coherence operator 18. The coherence correlations are then described by the equilibrium correlation function of $\tilde{\sigma}_{x}$,

$$
C_{x}(t)=\left\langle\tilde{\sigma}_{x}(t) \tilde{\sigma}_{x}(0)\right\rangle_{\beta}=\operatorname{Tr}\left[\tilde{\sigma}_{x}(t) \tilde{\sigma}_{x}(0) W_{\beta}\right],
$$

where $W_{\beta}=e^{-\beta H} / \operatorname{Tr}\left[e^{-\beta H}\right]$ is the equilibrium density matrix of the global system, and $\tilde{\sigma}_{x}(t)$ is the Heisenberg-representation of $\tilde{\sigma}_{x}$ with respect to the untransformed Hamiltonian 
(2.1) (cf. Ref. [19]). The associated response function $\chi_{x}(t)=(-2 / \hbar) \Theta(t) \operatorname{Im} C_{x}(t)$ describes the linear response of the system to a coherence inducing perturbation $H_{\text {pert }} \propto \tilde{\sigma}_{x}$.

It is convenient to consider $C_{x}(t)$ as the mean value of $\tilde{\sigma}_{x}(t)$ with respect to the "density matrix" $W=\tilde{\sigma}_{x}(0) W_{\beta}$. Switching to the Schrödinger-picture, Eq. (2.10) becomes

$$
C_{x}(t)=\operatorname{Tr}\left[\tilde{\sigma}_{x} W(t)\right]
$$

where the time-dependent "density matrix" $W(t)=\exp (-i H t / \hbar) W(0) \exp (i H t / \hbar)$ obeys the initial condition $W(0)=\tilde{\sigma}_{x} W_{\beta}$. Inserting the expression (2.8) for $\tilde{\sigma}_{x}$ into Eq. (2.11) and performing the trace, we find that $C_{x}(t)$ is the sum of the off-diagonal matrix elements

$$
C_{x}(t)=\rho_{1,-1}^{(s)}(t)+\rho_{-1,1}^{(-s)}(t)
$$

Since $\tilde{\sigma}_{x}$ acts also in the bath space, $\rho_{i, j}^{(s)}(t)$ is different from the usual reduced density matrix as it appears, e.g., in the $\sigma_{z}$ correlation function [5]. We shall refer to $\rho_{i, j}^{(s)}(t)$ as the "shifted reduced density matrix" (SRDM). To be general, we now give the discussion for a continuous variable $q$, and return to the two-state system only in Section IV. We have

$$
\begin{aligned}
\rho^{(s)}\left(q_{f}, q_{f}^{\prime}, t\right)= & \int d x_{f}\left\langle q_{f}, x_{f}+s|W(t)| q_{f}^{\prime}, x_{f}\right\rangle \\
= & \int d q_{i} d q_{i}^{\prime} d x_{f} d x_{i} d x_{i}^{\prime} K\left(q_{f}, x_{f}+s, t ; q_{i}, x_{i}, 0\right) \\
& \times K^{*}\left(q_{f}^{\prime}, x_{f}, t ; q_{i}^{\prime}, x_{i}^{\prime}, 0\right)\left\langle q_{i}, x_{i}|W(0)| q_{i}^{\prime}, x_{i}^{\prime}\right\rangle
\end{aligned}
$$

where $K\left(q_{f}, x_{f}+s, t ; q_{i}, x_{i}, 0\right)$ is the usual Feynman propagator that may be expressed as a path-integral. The matrix elements of the initial "density matrix" $W(0)=\tilde{\sigma}_{x} W_{\beta} \operatorname{read}$

$$
\left\langle q_{i}, x_{i}|W(0)| q_{i}^{\prime}, x_{i}^{\prime}\right\rangle=\left\langle-q_{i}, x_{i}-s \operatorname{sgn}\left(q_{i}\right)\left|W_{\beta}\right| q_{i}^{\prime}, x_{i}^{\prime}\right\rangle
$$

where the bath coordinates are also affected by the preparation. Now, it remains to integrate out the shifted bath degrees of freedom in the expression (2.13).

\section{GENERAL INITIAL PREPARATIONS}

The standard Feynman-Vernon approach which may be used to eliminate the bath degrees of freedom relies on the assumption of a factorized system-bath initial state. For ergodic systems, it is possible to obtain a real-time description also for a thermal initial state [2]. This approach can be generalized to special classes of correlated initial states by introducing a preparation function [20]. For the case of $C_{x}(t)$, the method in Ref. [20] has to be reconsidered, since the initial preparation also involves the bath. To proceed, we define a generalized preparation function $\lambda_{\mathrm{G}}\left(q_{i}, q_{i}^{\prime} ; \bar{q}, \bar{q}^{\prime} ; x_{i}, x_{i}^{\prime} ; \bar{x}, \bar{x}^{\prime}\right)$ by

$$
\left\langle q_{i}, x_{i}|W(0)| q_{i}^{\prime}, x_{i}^{\prime}\right\rangle=\int d \bar{q} d \bar{q}^{\prime} d \bar{x} d \bar{x}^{\prime} \lambda_{\mathrm{G}}\left(q_{i}, q_{i}^{\prime} ; \bar{q}, \bar{q}^{\prime} ; x_{i}, x_{i}^{\prime} ; \bar{x}, \bar{x}^{\prime}\right)\left\langle\bar{q}, \bar{x}\left|W_{\beta}\right| \bar{q}^{\prime}, \bar{x}^{\prime}\right\rangle .
$$

Comparing this form with Eq. (2.14), we see that the preparation function factorizes as

$$
\lambda_{\mathrm{G}}\left(q_{i}, q_{i}^{\prime} ; \bar{q}, \bar{q}^{\prime} ; x_{i}, x_{i}^{\prime} ; \bar{x}, \bar{x}^{\prime}\right)=\lambda_{\mathrm{S}}\left(q_{i}, q_{i}^{\prime} ; \bar{q}, \bar{q}^{\prime}\right) \lambda_{\mathrm{R}}\left(x_{i}, x_{i}^{\prime} ; \bar{x}, \bar{x}^{\prime}\right)
$$


where the system's and reservoir's preparation functions are given by

$$
\begin{aligned}
\lambda_{\mathrm{S}}\left(q_{i}, q_{i}^{\prime} ; \bar{q}, \bar{q}^{\prime}\right) & =\delta\left(q_{i}+\bar{q}\right) \delta\left(q_{i}^{\prime}-\bar{q}^{\prime}\right), \\
\lambda_{\mathrm{R}}\left(x_{i}, x_{i}^{\prime} ; \bar{x}, \bar{x}^{\prime}\right) & =\delta\left(x_{i}-\bar{x}-s \operatorname{sgn}\left(q_{i}\right)\right) \delta\left(x_{i}^{\prime}-\bar{x}^{\prime}\right) .
\end{aligned}
$$

With the form (3.2), the evolution of the SRDM (2.13) is given by

$$
\rho^{(s)}\left(q_{f}, q_{f}^{\prime}, t\right)=\int d q_{i} d q_{i}^{\prime} d \bar{q} d \bar{q}^{\prime} J_{\mathrm{G}}\left(q_{f}, q_{f}^{\prime}, t ; q_{i}, q_{i}^{\prime} ; \bar{q}, \bar{q}^{\prime}\right) \lambda_{\mathrm{S}}\left(q_{i}, q_{i}^{\prime} ; \bar{q}, \bar{q}^{\prime}\right)
$$

where the generalized propagating function reads

$$
\begin{aligned}
J_{\mathrm{G}}\left(q_{f}, q_{f}^{\prime}, t ; q_{i}, q_{i}^{\prime} ; \bar{q}, \bar{q}^{\prime}\right)= & \int d x_{f} d x_{i} d x_{i}^{\prime} d \bar{x} d \bar{x}^{\prime} K\left(q_{f}, x_{f}+s, t ; q_{i}, x_{i}, 0\right) \\
& \times\left\langle\bar{q}, \bar{x}\left|W_{\beta}\right| \bar{q}^{\prime}, \bar{x}^{\prime}\right\rangle K^{*}\left(q_{f}, x_{f}, t ; q_{i}, x_{i}, 0\right) \lambda_{\mathrm{R}}\left(x_{i}, x_{i}^{\prime} ; \bar{x}, \bar{x}^{\prime}\right) .
\end{aligned}
$$

For an ergodic system, the thermal density matrix $W_{\beta}$ in Eq. (3.6) can be expressed as follows. Describe the global system at a time $t_{0}<0$ by a factorized density matrix, the system being in a position eigenstate, say $\left|q_{0}\right\rangle$, and the reservoir being in thermal equilibrium, $W\left(t_{0}\right)=\left|q_{0}\right\rangle\left\langle q_{0}\right| \otimes e^{-\beta H_{\mathrm{R}}} / \operatorname{Tr}\left[e^{-\beta H_{\mathrm{R}}}\right]$ [2], and let it evolve out of this state under the full Hamiltonian. Then, if $t_{0}$ is sent to the infinite past, the system has reached at time zero the correlated initial state $\left\langle\bar{q}, \bar{x}\left|W_{\beta}\right| \bar{q}^{\prime}, \bar{x}^{\prime}\right\rangle$. With these considerations, we may rewrite (3.6) as

$$
\begin{aligned}
J_{\mathrm{G}}\left(q_{f}, q_{f}^{\prime}, t ; q_{i}, q_{i}^{\prime}, 0^{+} ; \bar{q}, \bar{q}^{\prime}, 0^{-} ; q_{0}, q_{0}^{\prime}, t_{0}\right) \\
\quad=\int \mathcal{D} q \int \mathcal{D} q^{\prime} \exp \left[\frac{i}{\hbar}\left(\mathcal{S}_{\mathrm{S}}[q]-\mathcal{S}_{\mathrm{S}}\left[q^{\prime}\right]\right)\right] \mathcal{F}_{\mathrm{G}}\left[q, q^{\prime} ; s\right]
\end{aligned}
$$

where $\mathcal{S}_{\mathrm{S}}[q]$ is the action corresponding to the system Hamiltonian (2.2). The functional integrations are over all paths $q\left(t^{\prime}\right)$ and $q^{\prime}\left(t^{\prime}\right)$ which satisfy the constraints

$$
\begin{aligned}
& q\left(t_{0}\right)=q_{0} \quad q\left(0^{-}\right)=\bar{q} \quad q\left(0^{+}\right)=q_{i} \quad q(t)=q_{f} \\
& q^{\prime}\left(t_{0}\right)=q_{0}^{\prime} \quad q^{\prime}\left(0^{-}\right)=\bar{q}^{\prime} \quad q^{\prime}\left(0^{+}\right)=q_{i}^{\prime} \quad q^{\prime}(t)=q_{f}^{\prime} .
\end{aligned}
$$

All the effects of the bath onto the system's dynamics are captured by the generalized influence functional

$$
\begin{aligned}
\mathcal{F}_{\mathrm{G}}\left[q, q^{\prime} ; s\right]= & \int d x_{f} d x_{i} d x_{i}^{\prime} d \bar{x} d \bar{x}^{\prime} d x_{0} d x_{0}^{\prime} W_{\mathrm{R}}\left(x_{0}, x_{0}^{\prime}\right) \lambda_{\mathrm{R}}\left(x_{i}, x_{i}^{\prime} ; \bar{x}, \bar{x}^{\prime}\right) \\
& \times \int \mathcal{D} x \int \mathcal{D} x^{\prime} \exp \left[\frac{i}{\hbar}\left(\mathcal{S}_{\mathrm{R}, \mathrm{I}}[x, q]-\mathcal{S}_{\mathrm{R}, \mathrm{I}}\left[x^{\prime}, q^{\prime}\right]\right)\right]
\end{aligned}
$$

where $\mathcal{S}_{\mathrm{R}, \mathrm{I}}[x, q]$ is the action corresponding to the reservoir and interaction terms in Eq. (2.1). The paths $x\left(t^{\prime}\right)$ and $x^{\prime}\left(t^{\prime}\right)$ are subject to the constraints

$$
\begin{aligned}
& x\left(t_{0}\right)=x_{0} \quad x\left(0^{-}\right)=\bar{x} \quad x\left(0^{+}\right)=x_{i} \quad x(t)=x_{f}+s \\
& x^{\prime}\left(t_{0}\right)=x_{0}^{\prime} \quad x^{\prime}\left(0^{-}\right)=\bar{x}^{\prime} \quad x^{\prime}\left(0^{+}\right)=x_{i} \quad x^{\prime}(t)=x_{f} .
\end{aligned}
$$

Compared to the standard Feynman-Vernon influence functional, there are two differences. First, the endpoint of the $x$-path is shifted by the displacement $s$, i.e., the bath does not end up in a diagonal state at time $t$. Second, the reservoir paths $x\left(t^{\prime}\right)$ and $x^{\prime}\left(t^{\prime}\right)$ are discontinous at time zero, depending on the reservoir's preparation function $\lambda_{\mathrm{R}}\left(x_{i}, x_{i}^{\prime} ; \bar{x}, \bar{x}^{\prime}\right)$. 


\section{EXACT FORMAL SOLUTION}

Having obtained an explicit expression for the SRDM at time $t$ for a general initial preparation, we can now write down the exact formal solution for the coherence correlations $C_{x}(t)$, Eq. (2.12). Inserting the preparation function (3.3) into the SRDM (3.5), we get

$$
C_{x}(t)=\lim _{t_{0} \rightarrow-\infty} \sum_{q_{i}, q_{i}^{\prime}, q_{f}} J_{\mathrm{G}}\left(q_{f},-q_{f}, t ; q_{i}, q_{i}^{\prime}, 0^{+} ;-q_{i}, q_{i}^{\prime}, 0^{-} ; q_{0}, q_{0}, t_{0}\right),
$$

where $q_{i}, q_{i}^{\prime}, q_{f}= \pm a / 2$. Note that there is a jump in the $q$-path at time zero. Inserting the reservoir preparation function (3.4) into the influence functional (3.10), the $x^{\prime}\left(t^{\prime}\right)$-path (3.12) turns out continuous at time $t^{\prime}=0$, whereas the $x\left(t^{\prime}\right)$-path (3.11) is discontinuous. Because of the integration over $x_{i}$, the constraints (3.11) may equivalently be expressed as

$$
x\left(t_{0}\right)=x_{0} \quad x\left(0^{-}\right)=x_{i} \quad x\left(0^{+}\right)=x_{i}+s \operatorname{sgn}\left(q_{i}\right) \quad x(t)=x_{f}+s \operatorname{sgn}\left(q_{f}\right) .
$$

Consider first the contributions to $C_{x}(t)$ with $q_{i}=q_{f}$. In this case, the shifts in the $x$-path at times $t^{\prime}=0^{+}$and $t^{\prime}=t$ are equal, and thus we can eliminate the shift at positive times by defining modified reservoir coordinates according to

$$
\tilde{x}\left(t^{\prime}\right)=x\left(t^{\prime}\right)-s \operatorname{sgn}\left(q_{i}\right) \Theta\left(t^{\prime}\right) .
$$

The path $\tilde{x}\left(t^{\prime}\right)$ is continuous at $t^{\prime}=0$ and obeys $\tilde{x}(t)=x_{f}$. In the shifted coordinate, the bath is in a diagonal state at time $t$. As the action $S_{\mathrm{R}, \mathrm{I}}[x, q]$ appearing in the influence functional is quadratic both in $x\left(t^{\prime}\right)$ and in $q\left(t^{\prime}\right)$ and bilinear in the coupling, the second term in Eq. (4.2) can be absorbed into a modified $q$-path which is continuous at $t^{\prime}=0$,

$$
\tilde{q}\left(t^{\prime}\right)=q\left(t^{\prime}\right)-2 q_{i} \Theta\left(t^{\prime}\right) .
$$

Writing the influence functional in terms of the paths $q^{\prime}\left(t^{\prime}\right), x^{\prime}\left(t^{\prime}\right)$ and the modified paths $\tilde{q}\left(t^{\prime}\right), \tilde{x}\left(t^{\prime}\right)$, the displacement $s$ is completely eliminated from the description. Thus, after integrating out the bath degrees of freedom, we end up with an influence functional that is of the standard Feynman-Vernon form for a factorized initial state at time $t_{0}$,

$$
\mathcal{F}_{\mathrm{G}}\left[q, q^{\prime} ; s \operatorname{sgn}\left(q_{i}\right)\right]=\mathcal{F}\left[\tilde{q}, q^{\prime}\right] .
$$

All effects in $\tilde{\sigma}_{x}$ induced by the polaronic cloud are in the modified path $\tilde{q}\left(t^{\prime}\right)$.

Next, consider the contributions to (4.1) with $q_{i}=-q_{f}$. Now, it is not possible to end up with an influence functional of the form (4.4) in which the shifts of the bath modes are fully absorbed into a modified path $\tilde{q}\left(t^{\prime}\right)$. In the usual charge picture (see below), the case

$q_{i}=-q_{f}$ corresponds to sequences of charges that violate overall neutrality. As a result, $\Delta$ and $\omega_{c}$ cannot be combined to a function of $\Delta_{r}$ alone. Instead, each contribution comes with an additional factor $\left(\Delta_{r} / \omega_{c}\right)^{4 K}$ and therefore is nonuniversal. Thus in the scaling limit $\Delta_{r} / \omega_{c} \rightarrow 0$, all contributions with $q_{i}=-q_{f}$ vanish.

With the above, the correlation function is now given by

$$
C_{x}(t)=\lim _{t_{0} \rightarrow-\infty} \sum_{q_{i}, q_{i}^{\prime}} J_{\mathrm{G}}\left(q_{i},-q_{i}, t ; q_{i}, q_{i}^{\prime}, 0^{+} ;-q_{i}, q_{i}^{\prime}, 0^{-} ; q_{0}, q_{0}, t_{0}\right) .
$$


At this stage, it is important to note that the free propagators in the propagating function depend on the original paths $q\left(t^{\prime}\right)$ and $q^{\prime}\left(t^{\prime}\right)$. The concept of modified paths is only used to express the generalized influence functional in the standard Feynman-Vernon form. For the evaluation of Eq. (4.5), it is convenient to introduce the linear combinations

$$
\begin{aligned}
& \eta(t)=\left[q(t)+q^{\prime}(t)\right] / a, \\
& \xi(t)=\left[q(t)-q^{\prime}(t)\right] / a,
\end{aligned}
$$

describing propagation along the diagonal of the density matrix and off-diagonal excursions, respectively. For the two-state system, these paths are piecewise constant with jumps at times $t_{j}$. As usual, the time intervals $t_{2 j}<t^{\prime}<t_{2 j+1}$ in which the system is in a diagonal state are called sojourns, while the time intervals $t_{2 j-1}<t^{\prime}<t_{2 j}$ spent in an off-diagonal state are referred to as blips. A sojourn is labelled by $\eta_{j}= \pm 1$, depending on whether the system is in state $R R$ or $L L$. Similarly, $\xi_{j}= \pm 1$ describes a blip in which the system is in state $R L$ or $L R$. The lengths of the sojourn- and blip-intervals are denoted by $s_{j}=t_{2 j+1}-t_{2 j}$ and $\tau_{j}=t_{2 j}-t_{2 j-1}$, respectively. All paths that contribute to the correlation function (4.5) start out from the initial sojourn $\eta_{0}$ at time $t_{0}$ and end in the blip state $\xi$ at time $t$. According to their behavior at time zero, they can be divided into two groups. In group A, the system jumps at time zero from a sojourn to a blip state $\left(q_{i}^{\prime}=-q_{i}\right.$ at time $\left.0^{+}\right)$. In group $\mathrm{B}$, the system hops at time zero from a blip to a sojourn state $\left(q_{i}^{\prime}=q_{i}\right.$ at time $\left.0^{+}\right)$. A general path with $n$ blips at negative and $m$ blips at positive times can be parametrized by

$$
\begin{aligned}
\eta\left(t^{\prime}\right) & =\sum_{j=0}^{n+m-1} \eta_{j}\left[\Theta\left(t^{\prime}-t_{2 j}\right)-\Theta\left(t^{\prime}-t_{2 j+1}\right)\right], \\
\xi\left(t^{\prime}\right) & =\sum_{j=1}^{n+m} \xi_{j}\left[\Theta\left(t^{\prime}-t_{2 j-1}\right)-\Theta\left(t^{\prime}-t_{2 j}\right)\right],
\end{aligned}
$$

with $t_{2 n+2 m}=t$ [21]. For group A, we have $t_{2 n+1}=0$, whereas $t_{2 n}=0$ for group B. According to the boundary conditions, the paths are subject to the constraints

$$
\begin{array}{ll}
\xi_{n+m}=\xi_{n+1}=-\eta_{n} & (\text { group A) } \\
\xi_{n+m}=-\xi_{n}=\eta_{n} & (\text { group B) } .
\end{array}
$$

Generic contributions to group A and group B are sketched in Fig. 11.

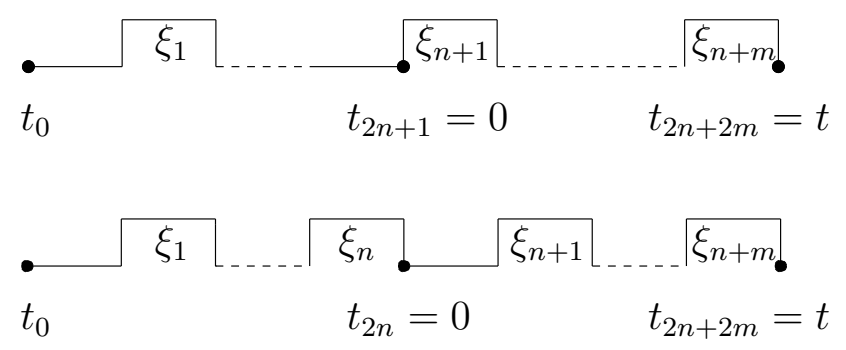

FIG. 1. Path contributions to group A (top) and group B (bottom). The steps represent blips of either sign, and sojourns are indicated by the baseline. 
Thus the correlation function is built up by two parts that correspond to these two different path classes. We have $C_{x}(t)=C_{x}^{\mathrm{A}}(t)+C_{x}^{\mathrm{B}}(t)$ with

$$
\begin{aligned}
C_{x}^{\mathrm{A}}(t) & =\lim _{t_{0} \rightarrow-\infty} \sum_{\xi} J_{\mathrm{G}}\left(\xi, t ; \xi, 0^{+} ; \eta=-\xi, 0^{-} ; \eta_{0}, t_{0}\right), \\
C_{x}^{\mathrm{B}}(t) & =\lim _{t_{0} \rightarrow-\infty} \sum_{\xi} J_{\mathrm{G}}\left(\xi, t ; \eta=\xi, 0^{+} ;-\xi, 0^{-} ; \eta_{0}, t_{0}\right) .
\end{aligned}
$$

The path sum is over all sequences of blips and sojourns and implies time-ordered integration over the jump times. We introduce the compact notation

$$
\int_{t_{0}}^{t} \mathcal{D}_{k, l}\left\{t_{j}\right\}=\int_{0}^{t} d t_{k+l+1} \int_{0}^{t_{k+l+1}} d t_{k+l} \ldots \int_{0}^{t_{k+3}} d t_{k+2} \int_{t_{0}}^{0} d t_{k} \ldots \int_{t_{0}}^{t_{2}} d t_{1} .
$$

Here $k$ and $l$ represent the number of flips in the time regimes $t_{0}<t^{\prime}<0$ and $0<t^{\prime}<t$, respectively. For group A, we have $k=2 n, l=2 m-2$, whereas for group $\mathrm{B} k=2 n-1$ and $l=2 m-1$. Each transition in Eq. (4.11) comes with a factor $\pm i \Delta / 2$. There are two additional transitions at time zero, $t_{k+1}=0$, and at time $t, t_{k+l+2}=t$. These two hops, however, come without a factor $\pm i \Delta / 2$ since they are not dynamical. The jump at time zero is enforced by the operation of $\tilde{\sigma}_{x}$, whereas the jump at time $t$ is introduced for convenience (cf. Ref. 21]). The amplitude to stay in a sojourn is unity, while the amplitude to stay in blip $\xi_{j}$ is given by $\exp \left(i \epsilon \xi_{j} \tau_{j}\right)$. Thus, a full path gives for both groups a factor

$$
-\eta_{0} \xi\left(-\Delta^{2} / 4\right)^{n+m-1} D_{n, m}
$$

with the bias term

$$
D_{n, m}=\exp \left(i \epsilon \sum_{j=1}^{n+m} \xi_{j} \tau_{j}\right)
$$

Before discussing the modifications due to the polaron transformation, consider the standard influence functional. Performing integrations by parts, it takes the form [2]

$$
\mathcal{F}[\eta, \xi]=\exp \left\{\int_{t_{0}}^{t^{+}} d t^{\prime} \int_{t_{0}}^{t^{\prime}} d t^{\prime \prime}\left[\dot{\xi}\left(t^{\prime}\right) S\left(t^{\prime}-t^{\prime \prime}\right) \dot{\xi}\left(t^{\prime \prime}\right)+i \dot{\xi}\left(t^{\prime}\right) R\left(t^{\prime}-t^{\prime \prime}\right) \dot{\eta}\left(t^{\prime \prime}\right)\right]\right\}
$$

where the kernels $S(t)$ and $R(t)$ are the real and imaginary part of the second integral of the bath correlation function. In the limit $\omega_{c} t \gg 1$, we have [2]

$$
\begin{aligned}
& S(t)=2 K \ln \left[\left(\hbar \beta \omega_{c} / \pi\right) \sinh (\pi t / \hbar \beta)\right], \\
& R(t)=\pi K \operatorname{sgn}(t) .
\end{aligned}
$$

Because of the form (4.7), the velocities in Eq. (4.14) consist of a series of delta-functions centered at the flip times. This suggests to regard the blip- and sojourn-paths as sequences of charges: blip-charges interact with each other through the kernel $S(t)$, while the sojourncharges interact with the blip-charges via $R(t)$. Substituting the paths (4.7) into Eq. (4.14), the influence functional takes the form

$$
\mathcal{F}_{n, m}=G_{n, m} H_{n, m}
$$


The factor $G_{n, m}$ contains all the inter- and intrablip-interactions,

$$
\begin{aligned}
G_{n, m} & =\exp \left[-\sum_{j=1}^{n+m} S_{2 j, 2 j-1}-\sum_{j=2}^{n+m} \sum_{k=1}^{j-1} \xi_{j} \xi_{k} \Lambda_{j, k}\right], \\
\Lambda_{j, k} & =S_{2 j, 2 k-1}+S_{2 j-1,2 k}-S_{2 j, 2 k}-S_{2 j-1,2 k-1},
\end{aligned}
$$

where $S_{p, q}=S\left(t_{p}-t_{q}\right)$. The sojourn-blip interactions are captured by the phase factor $H_{n, m}$. With the form (4.16), each sojourn only interacts with the subsequent blip,

$$
H_{n, m}=\exp \left[i \pi K \sum_{k=0}^{n+m-1} \eta_{k} \xi_{k+1}\right] \text {. }
$$

Substituting Eq. (4.15) into the term $\Delta^{2 n+2 m} G_{n, m}$, the quantities $\Delta$ and $\omega_{c}$ are combined into a factor $\Delta_{r}^{(2-2 K)(n+m)}$, where $\Delta_{r}$ is the renormalized tunneling frequency, Eq. (2.5). The autocorrelation function of the bare $\sigma_{x}$ depends on the standard influence functional (4.17)-(4.20). In this case, however, there appears the quantity $\Delta^{2 n+2 m-2} G_{n, m}$ because the $\Delta$-factors of the two blip-charges at time zero and time $t$ are missing. Therefore the autocorrelation function of the bare $\sigma_{x}$ comes with an overall factor $\Delta_{r}^{2} / \Delta^{2}=\left(\Delta_{r} / \omega_{c}\right)^{2 K}$. Thus it is nonuniversal in the sense discussed above.

Now return to the correlation function $C_{x}(t)$ which depends on the generalized influence functional $\mathcal{F}_{\mathrm{G}}$. As shown above, this can be expressed in the standard form (4.14) if we substitute the modified paths

$$
\begin{aligned}
& \tilde{\xi}\left(t^{\prime}\right)=\xi\left(t^{\prime}\right)-\xi\left[\Theta\left(t^{\prime}\right)-\Theta\left(t^{\prime}-t\right)\right] \\
& \tilde{\eta}\left(t^{\prime}\right)=\eta\left(t^{\prime}\right)-\xi\left[\Theta\left(t^{\prime}\right)-\Theta\left(t^{\prime}-t\right)\right] .
\end{aligned}
$$

The effects of the subtractions in Eq. (4.21) are directly seen in the charge picture. Taking into account the constraints (4.8) and (4.9), one gets the following changes: In the path $\tilde{\xi}\left(t^{\prime}\right)$, the two blip-charges at times $t^{\prime}=0$ and $t^{\prime}=t$ are cancelled. In the path $\tilde{\eta}\left(t^{\prime}\right)$, the sojourn-charge originally located at time $t^{\prime}=0$ is moved to time $t^{\prime}=t$. It turns out that the influence functionals for the paths of group A and group B are different. We write

$$
\mathcal{F}_{n, m}^{\mathrm{A}}=G_{n, m}^{\mathrm{A}} H_{n, m}^{\mathrm{A}}, \quad \mathcal{F}_{n, m}^{\mathrm{B}}=G_{n, m}^{\mathrm{B}} H_{n, m}^{\mathrm{B}} .
$$

The blip-interaction-factors $G_{n, m}^{\mathrm{A} / \mathrm{B}}$ differ from the standard $G_{n, m}$ by the absence of the two blip charges at $t^{\prime}=0$ and $t^{\prime}=t$. For group $\mathrm{A}$, this is

$$
G_{n, m}^{\mathrm{A}}=\exp \left[-\sum_{\substack{j=1 \\ j \neq n+1}}^{n+m} S_{2 j, 2 j-1}-\sum_{j=2}^{n+m} \sum_{k=1}^{j-1} \xi_{j} \xi_{k} \Lambda_{j, k}^{\mathrm{A}}\right],
$$

where $\Lambda_{j, k}^{\mathrm{A}}$ describes the interblip correlations for the modified sequence of charges. If $j, k \neq$ $n+1$ and $\neq n+m, \Lambda_{j, k}^{\mathrm{A}}$ is again given by (4.19). In all other cases, the interactions of the missing charges have to be dropped in (4.19). For instance, for $j=n+1$, we have $\Lambda_{n+1, k}^{\mathrm{A}}=S_{2 n+2,2 k-1}-S_{2 n+2,2 k}$. Similarly, we obtain for group B

$$
G_{n, m}^{\mathrm{B}}=\exp \left[-\sum_{\substack{j=1 \\ j \neq n}}^{n+m} S_{2 j, 2 j-1}-\sum_{j=2}^{n+m} \sum_{k=1}^{j-1} \xi_{j} \xi_{k} \Lambda_{j, k}^{\mathrm{B}}\right]
$$


with analogous modifications in $\Lambda_{j, k}^{\mathrm{B}}$ for $j, k=n$ and $n+m$. For instance, we have $\Lambda_{n, k}^{\mathrm{B}}=$ $S_{2 n-1,2 k}-S_{2 n-1,2 k-1}$. The modified phase factors $H_{n, m}^{i}$ take the form

$$
\begin{aligned}
H_{n, m}^{\mathrm{A}} & =\exp \left[i \pi K \sum_{\substack{k=0 \\
k \neq n}}^{n+m-1} \eta_{k} \xi_{k+1}\right] \\
H_{n, m}^{\mathrm{B}} & =\exp \left[i \pi K \sum_{\substack{k=0 \\
k \neq n}}^{n+m-1}\left(\eta_{k} \xi_{k+1}+\eta_{n}\left(\xi_{n+1}-\xi_{n+m}\right)\right)\right] .
\end{aligned}
$$

Thus each sojourn interacts with the subsequent blip except for sojourn $n$. For group A, the sojourn $n$ is effectively noninteracting, whereas for group B, it effectively interacts both with blip $n$ and blip $n+m$.

At this point, let us briefly reflect what we have gained so far. First of all, since the sequence of the remaining $2 n+2 m-2$ blip charges is neutral and comes with a factor $\Delta^{2(n+m-1)}$, the quantities $\Delta$ and $\omega_{c}$ are combined into a factor $\Delta_{r}^{(2-2 K)(n+m-1)}$. Thus, the $\tilde{\sigma}_{x}$ autocorrelation function turns out to be universal. There is, however, an essential difference between the two groups. For group A, the charges in the negative and positive time branches are neutral individually. For group B, there is an excess charge \pm 1 in each branch, and only the combined arrangement is neutral again. Since the asymptotic decay of equilibrium correlation functions crucially depends on the interactions between the negative and positive time branches, we should expect different behaviors for group A and group B.

Collecting the various results, we obtain explicit expressions for the propagating functions of group A and group B in Eq.(4.10),

$$
\begin{aligned}
& J_{\mathrm{G}}\left(\xi, t ; \xi, 0^{+} ; \eta=-\xi, 0^{-} ; \eta_{0}, t_{0}\right) \\
& \quad=-\eta_{0} \xi \sum_{n=0}^{\infty} \sum_{m=1}^{\infty}\left(-\frac{\Delta^{2}}{4}\right)^{n+m-1} \int_{t_{0}}^{t} \mathcal{D}_{2 n, 2 m-2}\left\{t_{j}\right\} \sum_{\left\{\xi_{j}\right\}^{\mathrm{A}}} G_{n, m}^{\mathrm{A}} D_{n, m} \sum_{\left\{\eta_{j}\right\}^{\mathrm{A}}} H_{n, m}^{\mathrm{A}}, \\
& J_{\mathrm{G}}\left(\xi, t ; \eta=\xi, 0^{+} ;-\xi, 0^{-} ; \eta_{0}, t_{0}\right) \\
& \quad=-\eta_{0} \xi \sum_{n=1}^{\infty} \sum_{m=1}^{\infty}\left(-\frac{\Delta^{2}}{4}\right)^{n+m-1} \int_{t_{0}}^{t} \mathcal{D}_{2 n-1,2 m-1}\left\{t_{j}\right\} \sum_{\left\{\xi_{j}\right\}^{\mathrm{B}}} G_{n, m}^{\mathrm{B}} D_{n, m} \sum_{\left\{\eta_{j}\right\}^{\mathrm{B}}} H_{n, m}^{\mathrm{B}} .
\end{aligned}
$$

The summation is over all $\xi_{j}, \eta_{j}= \pm 1$. The superscripts $\{\ldots\}^{\mathrm{A}}$ and $\{\ldots\}^{\mathrm{B}}$ indicate the constraints (4.8) and (4.9) with $\xi_{n+m}=\xi$, respectively. Using Eqs. (4.10), $C_{x}(t)$ is obtained. It is now straightforward to perform the $\eta$-summations and to use symmetry relations under exchange $\left\{\xi_{j}\right\} \rightarrow\left\{-\xi_{j}\right\}$. Taking the limit $t_{0} \rightarrow-\infty$, the correlation function becomes independent of the initial value $\eta_{0}$. In the end, we find for the symmetrized correlation function $S_{x}(t)=\operatorname{Re} C_{x}(t)$ and the response function $\chi_{x}(t)=(-2 / \hbar) \Theta(t) \operatorname{Im} C_{x}(t)$ the expressions 


$$
\begin{aligned}
S_{x}^{\mathrm{A}}(t)= & \frac{1}{2} \sum_{m=1}^{\infty}\left(-\bar{\Delta}^{2}\right)^{m-1} \int_{-\infty}^{t} \mathcal{D}_{0,2 m-2}\left\{t_{j}\right\} \sum_{\left\{\xi_{j}\right\}^{\mathrm{A}}} G_{0, m}^{\mathrm{A}} D_{0, m}^{(+)} \\
S_{x}^{\mathrm{B}}(t)= & -\sum_{n=1}^{\infty} \sum_{m=2}^{\infty}\left(-\bar{\Delta}^{2}\right)^{n+m-1} \sin ^{2}(\pi K) \int_{-\infty}^{t} \mathcal{D}_{2 n-1,2 m-1}\left\{t_{j}\right\} \sum_{\left\{\xi_{j}\right\}} \xi_{1} \xi_{n+m} G_{n, m}^{\mathrm{B}} D_{n, m}^{(+)}, \\
\chi_{x}^{\mathrm{A}}(t)= & \frac{1}{\hbar} \sum_{n=1}^{\infty} \sum_{m=1}^{\infty}\left(-\bar{\Delta}^{2}\right)^{n+m-1} \tan (\pi K) \int_{-\infty}^{t} \mathcal{D}_{2 n, 2 m-2}\left\{t_{j}\right\} \sum_{\left\{\xi_{j}\right\}^{\mathrm{A}}} \xi_{1} \xi_{n+m} G_{n, m}^{\mathrm{A}} D_{n, m}^{(+)} \\
\chi_{x}^{\mathrm{B}}(t)= & \frac{1}{\hbar} \sum_{n=1}^{\infty} \sum_{m=1}^{\infty}\left(-\bar{\Delta}^{2}\right)^{n+m-1} \tan (\pi K) \int_{-\infty}^{t} \mathcal{D}_{2 n-1,2 m-1}\left\{t_{j}\right\} \\
& \times \sum_{\left\{\xi_{j}\right\}^{\mathrm{B}}} \xi_{1} G_{n, m}^{\mathrm{B}} D_{n, m}^{(+)}\left\{\sin ^{2}(\pi K) \xi_{n+1}+\cos ^{2}(\pi K) \xi_{n+m}\right\}
\end{aligned}
$$

Here we have introduced $\bar{\Delta}^{2}=\Delta^{2} \cos (\pi K) / 2$ and

$$
D_{n, m}^{(+)}=\cos \left(\epsilon \sum_{j=1}^{n+m} \xi_{j} \tau_{j}\right)
$$

Equations (4.28)-(4.32) are exact formal series expansions for the symmetrized equilibrium correlation function $S_{x}(t)$ and the response function $\chi_{x}(t)$. Despite their formidable appearance, we can obtain exact results in certain limits. This is discussed in the remainder of this work. For the subsequent analysis, it is convenient to switch from integrations over the flip times, Eq. (4.11), to integrations over blip lengths $\tau_{j}$ and sojourn lengths $s_{j}$.

\section{THE CASE $K=\frac{1}{2}$}

For the value $K=\frac{1}{2}$, the above series for $S_{x}(t)$ and $\chi_{x}(t)$ can be summed in analytical form using the concept of collapsed blips and collapsed sojourns [5]. Putting $K=\frac{1}{2}-\kappa$ with $\kappa \ll 1$, the phase factor $\cos (\pi K) \approx \pi \kappa$ vanishes in the limit $\kappa \rightarrow 0$. In order to have a finite contribution for $K=\frac{1}{2}$, each factor $\cos (\pi K)$ has to be compensated by a $1 / \kappa$ singularity arising from the "short-distance" singularity of the breathing mode integral of a dipole (blip or sojourn) with interaction $e^{-S(\tau)} \approx\left(\omega_{c} \tau\right)^{-(1-2 \kappa)}$. Thus we have

$$
I\left(K=\frac{1}{2}\right)=\lim _{K \rightarrow 1 / 2} \Delta^{2} \cos (\pi K) \int_{0} d \tau e^{-S(\tau)}=\frac{\pi}{2} \frac{\Delta^{2}}{\omega_{c}} \equiv \gamma .
$$

We shall refer to an expression of the form (5.1) as a collapsed dipole. Since a collapsed dipole has zero dipole moment, it does not interact with other charges. Further, it is insensitive to a symmetric bias factor. In contrast, an odd bias factor in Eq. (5.1) prevents a dipole from collapsing, and combined with a factor $\cos (\pi K)$, this term vanishes as $K \rightarrow \frac{1}{2}$. 
A blip or a sojourn becomes extended when the $\cos (\pi K)$ factor and the short-distance singularity are absent. Within an extended blip of length $\tau$, the system may make any number of visits of duration zero to a sojourn and then returns to the same blip. Mathematically, this is described by the insertion of a grand-canonical ensemble of noninteracting collapsed sojourns (CS), yielding a CS form factor $e^{-\gamma \tau / 2}$. Likewise, within a sojourn of length $s$, the system may make any number of visits of duration zero to a blip state. This is represented by a grand-canonical ensemble of noninteracting collapsed blips (CB). Since the system can return to either sojourn state, there is a multiplicity factor 2, yielding a CB form factor $e^{-\gamma s}$.

An extended sojourn, say $s_{k}$, remains free of insertions only if the subsequent blip is weighted with an unconstrained factor $\xi_{k+1}$. In this case, the $\left\{\xi_{j}\right\}$ summation leads to cancellations among the interactions stretching over the extended sojourn, and thus it remains bare. It turns out that this is a general rule also for $K \neq \frac{1}{2}$, referred to as the $\xi$-rule in the sequel. For the correlation functions (4.29)-(4.31), e.g., the initial sojourn starting at $t_{0}$ remains bare due to the factor $\xi_{1}$ in the exact formal expressions. There are no other bare intervals in the negative-time branch. Thus, the limit $t_{0} \rightarrow-\infty$ is well-behaved.

Based on these concepts, we now analyze the various contributions. Consider first the symmetrized correlation function. Assigning the $\cos (\pi K)$ factors to the collapsing dipoles as in Eq. (5.1), there is one $\cos (\pi K)$ factor left in Eq. (4.29). Thus the contribution $S_{x}^{\mathrm{B}}(t)$ vanishes linearly with $\kappa$ as $K \rightarrow \frac{1}{2}$. In $S_{x}^{\mathrm{A}}(t)$, the system dwells in the initial sojourn state $\eta$ until time zero. At this time, it hops into the blip state $\xi=-\eta$ where it stays until time $t$, resulting in a factor $\cos (\epsilon t)$. The blip of length $t$ is decorated with a CS form factor. Piecing the various components together, we find the damped oscillatory behavior

$$
S_{x}(t)=S_{x}^{\mathrm{A}}(t)=\cos (\epsilon t) e^{-\gamma t / 2} .
$$

The contributions to $S_{x}^{\mathrm{A}}(t)$ are sketched diagrammatically in Fig. 2. Since only collapsed sojourns contribute to $S_{x}(t)$ and the short-distance behavior of the pair interaction is independent of temperature, the expression (5.2) is valid at any temperature.

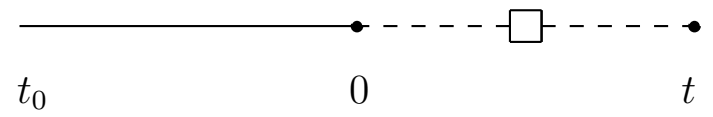

FIG. 2. The diagram describing $S_{x}(t)$. The full and dashed lines represent sojourns and blips, respectively. The empty box represents the insertion of a CS form factor within the blip interval. The bullets mark transitions which are free of bath correlations because of the modified influence functional.

Consider next the response function. The contribution of group A is sketched in Fig. 3. In the negative-time branch, the initial sojourn is followed by an extended blip and an extended sojourn state. Both of them are equipped with a CS and CB form factor, respectively. At time zero, the system hops back into a blip state and stays there until time $t$. The extended blip is again decorated with a CS form factor. In mathematical terms, we have for $t>0$

$$
\chi_{x}^{\mathrm{A}}(t)=\frac{2}{\hbar} \Delta^{2} \sin (\epsilon t) e^{-\gamma t / 2} \int_{0}^{\infty} d \tau d s \sin (\epsilon \tau) e^{-S(\tau)} e^{-\gamma \tau / 2} e^{-\gamma s} .
$$


Now, as shown in Ref. [5], the double integral times the factor $\Delta^{2}$ is just $P_{\infty}=\left\langle\sigma_{z}(t \rightarrow \infty)\right\rangle$. In the end, we find

$$
\begin{aligned}
\chi_{x}^{\mathrm{A}}(t) & =(2 / \hbar) P_{\infty} \sin (\epsilon t) e^{-\gamma t / 2}, \\
P_{\infty} & =\frac{2}{\pi} \operatorname{Im} \psi\left(\frac{1}{2}+\frac{\hbar \gamma}{4 \pi k_{\mathrm{B}} T}+i \frac{\hbar \epsilon}{2 \pi k_{\mathrm{B}} T}\right),
\end{aligned}
$$

where $\psi(z)$ is Euler's digamma function. Thus we find again exponential decay, resulting from exponential suppression factors due to collapsed blips or sojourns in each interval.

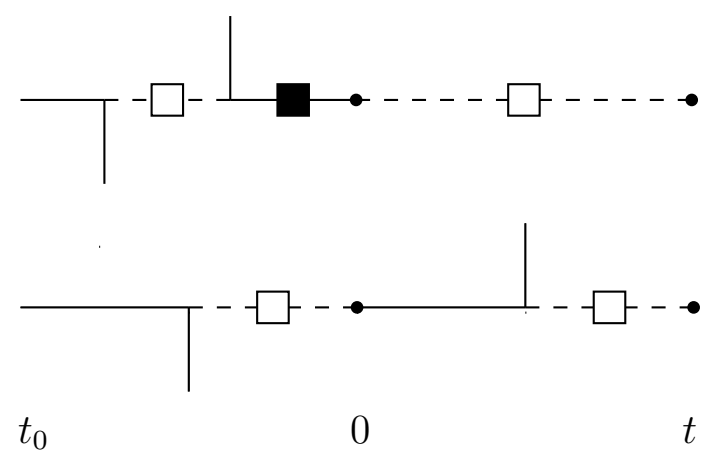

FIG. 3. The diagrams for $\chi_{x}^{\mathrm{A}}(t)$ (top) and $\chi_{x}^{\mathrm{B}}(t)$ (bottom). The full box represents the insertion of a CB form factor. The other symbols are analogous to Fig. 2. The upward and downward spikes symbolize the remaining charges.

Now we turn to the contributions of group B. It is immediately clear that the part of $\chi_{x}^{\mathrm{B}}(t)$ resulting from the second term in the curly bracket of Eq. (4.31) vanishes as $\kappa^{2}$ in the limit $\kappa \rightarrow 0$, whereas the first term is nonzero in this limit. Here, the system hops from the initial sojourn into a blip at a negative time $-\tau$ and stays there until time zero, where it returns to a sojourn state. At time $s$ it hops again into a blip state and dwells in this state until time $t$. Again, each blip interval is decorated with a CS form factor, as discussed above. Because of the factor $\xi_{n+1}$ in Eq. (4.31), however, the extended sojourn in the positive time branch is free of collapsed blips. The interacting dipole has length $\tau+s$ and introduces correlations between the negative and positive time branches (see Fig. 3). Thus we have

$$
\chi_{x}^{\mathrm{B}}(t)=\frac{\Delta^{2}}{\hbar} \int_{0}^{\infty} d \tau \int_{0}^{t} d s e^{-\gamma(t+\tau-s) / 2} e^{-S(\tau+s)} \cos [\epsilon(t-\tau-s)] .
$$

Introducing the dipole length $\tau+s$ as a new integration variable, performing the other integrations, and combining the resulting expression with Eq. (5.4), we obtain

$$
\chi_{x}(t)=(2 / \hbar)\left[\sin (\epsilon t) F_{1}(t)+\cos (\epsilon t) F_{2}(t)\right]
$$

with the functions

$$
\begin{aligned}
& F_{1}(t)=\frac{\Delta^{2}}{2 \gamma} \int_{0}^{\infty} d \tau e^{-S(\tau)} \sin (\epsilon \tau)\left(e^{-\gamma|t-\tau| / 2}+e^{-\gamma(t+\tau) / 2}\right), \\
& F_{2}(t)=\frac{\Delta^{2}}{2 \gamma} \int_{0}^{\infty} d \tau e^{-S(\tau)} \cos (\epsilon \tau)\left(e^{-\gamma|t-\tau| / 2}-e^{-\gamma(t+\tau) / 2}\right) .
\end{aligned}
$$


For asymptotic times $t \gg 1 / \gamma$, we find from Eq. (5.7) at zero temperature

$$
\chi_{x}(t)=\frac{8}{\pi \hbar} \frac{\gamma^{2}}{\gamma^{2}+4 \epsilon^{2}} \frac{1}{\gamma t} .
$$

The algebraic decay law arises from the contribution of group B. Because of the absence of collapsed blips in the sojourn interval $s$, this interval gets effectively very large, $s \approx t$ for $t \gg 1 / \gamma$. The $1 / t$ law in Eq. (5.10) is simply the signature of the bare intra-dipole interaction, $e^{-S(t)} \propto 1 / t$ for $K=\frac{1}{2}$. The algebraic law at $T=0$ is not only of academic interest, since it is also valid at low but finite temperatures in the intermediate time regime $1 / \gamma \ll t \ll \hbar \beta$. In the asymptotic limit $t \gg \hbar \beta \gg 1 / \gamma$, we find exponential decay,

$$
\chi_{x}(t)=\frac{16}{\hbar \gamma} \frac{\gamma^{2}}{\gamma^{2}+4 \epsilon^{2}} \frac{1}{\hbar \beta} e^{-\nu_{1} t / 2}
$$

where $\nu_{1}=2 \pi / \hbar \beta$ is the lowest bosonic Matsubara frequency.

Since we have calculated the expressions (5.2) and (5.7) independently, we are now in a position to verify whether they are consistent with the fluctuation-dissipation theorem,

$$
S_{x}(\omega)=\hbar \operatorname{coth}(\hbar \beta \omega / 2) \chi_{x}^{\prime \prime}(\omega) .
$$

Taking the Fourier transform of $S_{x}(t)$ and of $\chi_{x}(t)$, we find for the spectral function $S_{x}(\omega)$ and the absorptive part of the dynamical coherence susceptibility $\chi_{x}(\omega)$

$$
\begin{aligned}
S_{x}(\omega) & =\gamma \frac{\gamma^{2} / 4+\omega^{2}+\epsilon^{2}}{\left(\gamma^{2} / 4+\omega^{2}+\epsilon^{2}\right)^{2}-4 \epsilon^{2} \omega^{2}}, \\
\chi_{x}^{\prime \prime}(\omega) & =\frac{\gamma}{\hbar} \tanh \left(\frac{\hbar \omega}{2 k_{\mathrm{B}} T}\right) \frac{\gamma^{2} / 4+\omega^{2}+\epsilon^{2}}{\left(\gamma^{2} / 4+\omega^{2}+\epsilon^{2}\right)^{2}-4 \epsilon^{2} \omega^{2}} .
\end{aligned}
$$

This confirms that the FDT is satisfied. Finally, the real part $\chi_{x}^{\prime}(\omega)$ of the dynamical susceptibility reads (we put $\epsilon=0$ for simplicity)

$$
\chi_{x}^{\prime}(\omega)=\frac{8 \gamma}{\pi \hbar} \frac{1}{\gamma^{2}+4 \omega^{2}} \operatorname{Re}\left[\psi\left(\frac{1}{2}+\frac{\hbar \gamma}{4 \pi k_{\mathrm{B}} T}\right)-\psi\left(\frac{1}{2}+i \frac{\hbar \omega}{2 \pi k_{\mathrm{B}} T}\right)\right] .
$$

The linear static susceptibility $\chi_{x}^{(0)}=\chi_{x}^{\prime}(\omega \rightarrow 0)$ diverges logarithmically as $T \rightarrow 0$.

One final remark on the case $K=\frac{1}{2}$ is appropriate. The correlation functions can also be calculated in a fermionic representation by exploiting the equivalence of the spinboson model for $K=\frac{1}{2}$ with the Toulouse limit of the anisotropic Kondo or resonance level model [2]. One finds that the $\sigma_{x}$ correlation function in the resonance level model directly corresponds to the $\tilde{\sigma}_{x}$ correlation function of the spin-boson model [19]. The investigation of the spin-boson model is convenient when we depart from the particular case $K=\frac{1}{2}$.

VI. THE CASE $K=\frac{1}{2}-\kappa$

\section{A. Expansion around $K=\frac{1}{2}$}

In the previous section, we have solved the case $K=\frac{1}{2}$ in analytic form by using the concept of collapsed blips and collapsed sojourns. Let us now consider the regime $K=\frac{1}{2}-\kappa$ 
with $\kappa \ll 1$ and perform an expansion around the solution of the correlation function for $K=\frac{1}{2}$. For finite $\kappa$, a dipole is actually no longer collapsed. The basic idea now is to develop a $\kappa$-expansion by systematically taking into account the finite lengths of the blips and sojourns. To put up a general computation scheme, it is essential to split the breathing mode integral $I(K)$ given in Eq. (5.1) into a contribution $I_{1}(K)$ from the short-length interval $0<\tau<1 / \tilde{\gamma}$ and a residual contribution $I_{2}(K)$ from lengths $\tau>1 / \tilde{\gamma}$. The inverse time scale $\tilde{\gamma}$ is selfconsistently determined by the short-time part $I_{1}(K)$. We have

$$
I_{1}\left(K=\frac{1}{2}-\kappa\right) \equiv \tilde{\gamma}=\Delta^{2} \pi \kappa \int_{0}^{1 / \tilde{\gamma}} d \tau \frac{1}{\left(\omega_{c} \tau\right)^{1-2 \kappa}} \approx \gamma\left(\frac{\omega_{c}}{\gamma}\right)^{2 \kappa}
$$

where the factor $\pi \kappa$ is the remnant of the $\cos (\pi K)$ phase factor. The frequency $\tilde{\gamma}$ is the effective inverse time scale of the problem. The short-time part $I_{1}(K)$, representing either a collapsed blip or a collapsed sojourn, can be treated exactly in the same manner as described in the previous section. That is, all possible arrangements of collapsed dipoles within an extended sojourn and blip of length $s$ and $\tau$, respectively, add up to a CB form factor $e^{-\tilde{\gamma} s}$ and $\mathrm{CS}$ form factor $e^{-\tilde{\gamma} \tau / 2}$. The strategy is then completed by developing a systematic scheme to calculate the contributions from the extended blip and sojourn intervals, $\tau_{j}, s_{j}>1 / \tilde{\gamma}$. Since the leading contribution $I_{2}(K)$ is of order $\kappa$, it is natural to set up an expansion of the correlation function in the number of extended dipoles. Clearly, this is not a systematic expansion in $\kappa$ since every extended dipole also contributes higher-order corrections in $\kappa$. However, the strategy will allow to extract the actual long-time behavior of the correlation function. To perform the analysis, it is useful to introduce a diagrammatic picture. A generic contribution of order $\kappa^{m}$ is obtained by adding $m$ extended dipoles to the respective diagram for $K=\frac{1}{2}$. Its structure and asymptotic behavior are essentially determined by the following rules which are drawn from the exact formal expressions.

1.) Insertion of an extended sojourn into a dressed blip interval leads to the diagram of Fig. 1 (top), whereas insertion of an extended blip into a dressed sojourn interval is diagrammatically represented in Fig. 1 (bottom).

2.) An extended dipole with insertion of a CB or CS form factor has an effective length of order $1 / \tilde{\gamma}$ and therefore cannot produce algebraic decay of the correlation function.

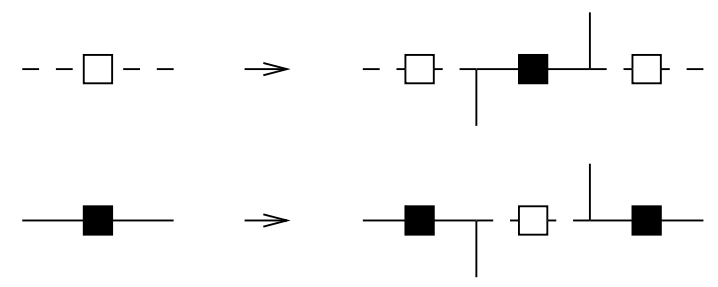

FIG. 4. Insertion of extended dipoles according to rule 2.

3.) An extended dipole which is free of CB and CS form factors has a length $\tau>>1 / \tilde{\gamma}$. Therefore, it is sensitive to the unscreened dipole interaction $e^{-S(\tau)} \propto \tau^{-2 K}$, and its length is eventually limited by the overall length $t$.

These rules are in correspondence with the above $\xi$-rule. We will now apply them to the symmetrized correlation function $S_{x}(t)$ and to the response function $\chi_{x}(t)$. 


\section{B. Response function $\chi_{x}(t)$}

We start the discussion of the response function by considering the path contributions of group A, Eq. (4.30). In order $\kappa^{m}$, the diagram for $\chi_{x}^{\mathrm{A}}(t)$, Fig. 3 (top), is supplemented by $m$ extended dipoles. They can be arbitrarily distributed among the negative and positive time branches using rule 1 (cf. Fig. (4). As a result, each interval (except for the first sojourn) is dressed by a CB or CS form factor. Thus, using argument $2, \chi_{x}^{\mathrm{A}}(t)$ decays exponentially.

Consider next the contribution to $\chi_{x}^{\mathrm{B}}(t)$ from the first term in the curly bracket of Eq. (4.31), referred to as $\chi_{x}^{\mathrm{B}_{1}}(t)$. In order $\kappa^{m}$, the diagram in Fig. 3 (bottom) for $\chi_{x}^{\mathrm{B}}(t)$ is modified as follows. There are insertions of $m$ extended sojourns which can be arbitrarily distributed among the two blip intervals displayed [rule 1]. Each of them is again confined to a length of order $1 / \tilde{\gamma}$. Due to the factor $\xi_{n+1}$ in Eq. (4.31), however, the initial sojourn at positive times remains free of insertions. At times $t \gg 1 / \tilde{\gamma}$, the length of this interval is therefore effectively $t$. Employing argument 3 , we see that the contribution $\chi_{x}^{\mathrm{B}_{1}}(t)$ decays as $e^{-S(t)} \propto t^{-(1-2 \kappa)}$. This law is generally valid, only the prefactor depends on the number of extended dipoles considered.

Starting with order $\kappa^{2}$, there is also a contribution from the second term in the curly bracket of (4.31), called $\chi_{x}^{\mathrm{B}_{2}}(t)$. The diagrams are as for $\chi_{x}^{\mathrm{B}_{1}}(t)$, apart from the crucial difference that the first sojourn in the positive time branch is dressed. This is due to the absence of the factor $\xi_{n+1}$ in $\chi_{x}^{\mathrm{B}_{2}}(t)$. Thus, according to rule $2, \chi_{x}^{\mathrm{B}_{2}}(t)$ decays exponentially.

\section{Symmetrized correlation function $S_{x}(t)$}

Consider first $S_{x}^{\mathrm{A}}(t)$, Eq. (4.28), which has dynamics only in the positive time branch. Employing rule 1, we have $m$ extended sojourns in order $\kappa^{m}$, and each of the blip and sojourn intervals is dressed. Thus, $S_{x}^{\mathrm{A}}(t)$ decays exponentially on the time scale $1 / \tilde{\gamma}$.

As emphasized in the previous section, the leading contribution to $S_{x}^{\mathrm{B}}(t)$ is of order $\kappa$. This term is found to be

$$
S_{x}^{\mathrm{B}}(t)=-\pi \kappa \frac{\Delta^{2}}{2} \int_{0}^{\infty} d \tau \int_{0}^{t} d s e^{-S(\tau+s)} e^{-\tilde{\gamma}(t+\tau-s) / 2}\left\{e^{-\tilde{\gamma} s}-1\right\} \cos [\epsilon(t-\tau-s)] .
$$

The reason for the subtraction in the curly bracket is the missing of diagrams without any insertions in the sojourn interval $s$. There is always at least one collapsed blip due to the constraint in the $\xi$-summation of expression (4.29). Introducing the length $\tau+s$ as a new integration variable, the other integrations can be performed. With the definition

$$
F_{3}(t)=\frac{\Delta^{2}}{2 \tilde{\gamma}} \int_{0}^{\infty} d \tau e^{-S(\tau)} \sin (\epsilon \tau)\left(e^{-\tilde{\gamma}|t-\tau| / 2}-e^{-\tilde{\gamma}(t+\tau) / 2}\right)
$$

and with $\gamma$ replaced by $\tilde{\gamma}$ in the expression (5.9) for $F_{2}(t)$, we find

$$
\begin{aligned}
S_{x}^{\mathrm{B}}(t)=\pi \kappa & \left\{\left[\cos (\epsilon t) F_{2}(t)+\sin (\epsilon t) F_{3}(t)\right]\right. \\
& -\frac{\Delta^{2}}{2}\left(\int_{0}^{t} d \tau \tau e^{-S(\tau)} e^{-\tilde{\gamma}(\tau+t) / 2} \cos [\epsilon(t-\tau)]\right. \\
& \left.\left.+t \int_{t}^{\infty} d \tau e^{-S(\tau)} e^{-\tilde{\gamma}(\tau+t) / 2} \cos [\epsilon(t-\tau)]\right)\right\} .
\end{aligned}
$$


The dominating contribution for $t \gg 1 / \tilde{\gamma}$ comes from the first line in Eq. (6.4), yielding

$$
S_{x}^{\mathrm{B}}(t)=4 \kappa \frac{\tilde{\gamma}^{2}}{\tilde{\gamma}^{2}+4 \epsilon^{2}}\left(\frac{1}{\tilde{\gamma} t}\right)^{1-2 \kappa} .
$$

The origin of the algebraic decay for $\kappa \neq 0$ is the subtraction term in the curly bracket in Eq. (6.2). The analysis shows that the subtraction also appears in all higher orders in $\kappa$. Thus the asymptotic behavior $t^{-(1-2 \kappa)}$ is generally valid for $\kappa \neq 0$. For $\kappa \rightarrow 0$, the prefactor of the algebraic decay law vanishes and the decay is exponential [cf. Eq. (5.2)].

\section{LONG-TIME BEHAVIOR FOR GENERAL $K<1$}

From the structure of the various contributions for $K=\frac{1}{2}-\kappa$, we can draw conclusions for the long-time behavior of the correlation functions for general $K<1$. For $K$ substantially different from $\frac{1}{2}$, the modifications concern the CB and CS form factors inserted in a given interval. Instead of collapsed noninteracting blips and sojourns, we now have extended interacting dipoles and it is no longer possible to perform the grand-canonical sum in analytic form. However, according to the $\xi$-rule, the sequences of charges are grouped into clusters which are separated by bare sojourns. Because of the alternating sum of the charges within a cluster, the length of a cluster is effectively of order $1 / \Delta_{r}$, where $\Delta_{r}$ is the renormalized frequency (2.5).

With this being the only essential modification, the asymptotic behaviors of the various contributions to the correlation function at times $t \gg 1 / \Delta_{r}$ emerge as follows. In group A, there is a single neutral cluster surrounding the origin of the time axis. Hence, both $S_{x}^{\mathrm{A}}(t)$ and $\chi_{x}^{\mathrm{A}}(t)$ decay exponentially. In group $\mathrm{B}$, we have a charged cluster in each time branch, satisfying overall neutrality. Since in both branches the initial sojourn is free of insertions, the two clusters are near the origin and near $t$, respectively, and they interact with the unscreened charge-charge interaction $e^{-S(t)} \propto t^{-2 K}$ [22]. This interaction directly determines the long-time behavior of $S_{x}^{\mathrm{B}}(t)$ and $\chi_{x}^{\mathrm{B}}(t)$. The contributions of group B predominate over the exponential contributions of group A for $t \gg 1 / \Delta_{r}$. Thus we have asymptotically

$$
\begin{aligned}
& S_{x}(t) \propto e^{-S(t)} \propto t^{-2 K}, \quad K \neq \frac{1}{2} \\
& \chi_{x}(t) \propto e^{-S(t)} \propto t^{-2 K} .
\end{aligned}
$$

Thus, the $\tilde{\sigma}_{x}$ autocorrelation function at $T=0$ decays with a power law. The power depends on the damping strength. Again, the $T=0$ decay laws (7.1) and (7.2) hold also at very low temperatures in the intermediate time regime $1 / \Delta_{r} \ll t \ll \hbar \beta$. In the asymptotic limit $t \gg \hbar \beta \gg 1 / \Delta_{r}$, the correlation functions show exponential decay,

$$
S_{x}(t) \propto e^{-K \nu_{1} t}, \quad \chi_{x}(t) \propto e^{-K \nu_{1} t},
$$

where the decay rate is $K$ times the lowest bosonic Matsubara frequency $\nu_{1}=2 \pi / \hbar \beta$.

Let us now put the decay law (7.1) in perspective with the generalized Shiba relation for the $\sigma_{z}$ correlation function [6 8, 12]. In the regime $t \gg 1 / \Delta_{r}$, this relation is expressed as

$$
S_{z}(t)=-2 K\left[\hbar \chi_{z}^{(0)} / 2\right]^{2} \frac{1}{t^{2}} .
$$


In a charge representation for $S_{z}(t)$, the $1 / t^{2}$ decay law reflects the dipole-dipole interaction between a neutral cluster in the negative-time branch and a neutral cluster in the positive time branch. The power of the algebraic interaction is independent of the coupling strength and is 2 for Ohmic dissipation.

Our findings are consistent with the fluctuation-dissipation theorem, Eq. (5.12). Upon Fourier transforming Eq. (7.2), we get $\chi_{x}^{\prime \prime}(\omega \rightarrow 0) \propto \operatorname{sgn}(\omega)|\omega|^{2 K-1}$ for $0<K<1$. Using the FDT relation (5.12) for $T=0$, we obtain $S_{x}(\omega \rightarrow 0)$, and transforming back to time, we find consistency with the law (7.1) for $S_{x}(t)$. As a by-product, we obtain a useful relation directly connecting the prefactors of the expressions (7.1) and (77.2),

$$
S_{x}(t)=(\hbar / 2) \cot (\pi K) \chi_{x}(t), \quad t \gg 1 / \Delta_{r} .
$$

In lowest order in $\kappa$, this relation is confirmed by the results (5.10) and (6.5). The case $K=\frac{1}{2}$ is special, since the prefactor of the $1 / t$ law for $S_{x}(t)$ vanishes according to the relation (7.5). This is in agreement with the result (6.5) obtained from the direct computation of $S_{x}(t)$.

The asymptotic decay law (7.2) leads to a different behavior for the linear (zero bias) static susceptibility $\chi_{x}^{(0)}=\int_{0}^{\infty} d t \chi_{x}(t)$ for $K$ below and above $\frac{1}{2}$. For $K<\frac{1}{2}$, the slow decay of $\chi_{x}(t)$ implies that the linear static susceptibility diverges algebraically, $\chi_{x}^{(0)} \propto T^{2 K-1}$ as $T \rightarrow 0$. This indicates that the system responds to a coherence inducing perturbation $\propto \tilde{\sigma}_{x}$ in a nonlinear manner. Interestingly, the regime $K<\frac{1}{2}$ coincides with the coherence regime for the population $\left\langle\sigma_{z}(t)\right\rangle$ at zero bias [10,15]. For $K>\frac{1}{2}$, the decay of $\chi_{x}(t)$ is sufficiently fast so that the linear static susceptibility is finite at $T=0$. This corresponds to the incoherent regime for $\left\langle\sigma_{z}(t)\right\rangle$ at zero bias. The transition from nonlinear to linear response

at $K=\frac{1}{2}$ thus reflects the intrinsic coherence properties of the system. These properties of the static susceptibility have been confirmed numerically in Ref. [15].

In conclusion, we have studied within a real-time approach the equilibrium correlation function of the polaron-dressed tunneling or coherence operator in the dissipative two-state system. This quantity turns out to be universal in the scaling limit. The elimination of the bath modes leads to a modified influence functional that can be recast into the standard form at the expense of introducing modified system paths. We have obtained the exact formal expressions for the coherence correlations for arbitrary damping strength $K$, and we have presented analytic results for the particular case $K=\frac{1}{2}$ and for the narrow regime $K=\frac{1}{2}-\kappa$ with $\kappa \ll 1$. The long-time behavior is found to be $\propto t^{-2 K}$ for general $K<1$, reflecting the loss of coherence with increasing damping strength. Generally, an algebraic decay law reveals that the initial state of the global system is correlated. In a charge picture, the particular decay $\propto t^{-2 K}$ expresses the interaction between two clusters with an excess charge of opposite sign. Since they are embedded in the vacuum ( $\xi$-rule), the interaction is unscreened.

\section{ACKNOWLEDGMENTS}

E.P. thanks R. Fazio and G. Giaquinta for useful discussions and acknowledges financial support by the INFM under the PRA-QTMD programme. Partial support was provided by the Sonderforschungsbereich 382 of the Deutsche Forschungsgemeinschaft (Bonn). 
[1] A.J. Leggett, S. Chakravarty, A.T. Dorsey, M.P.A.Fisher, A. Garg, and W. Zwerger, Rev. Mod. Phys. 59, 1 (1987); ibid. 67, 725 (1995) [erratum].

[2] U. Weiss, Quantum Dissipative Systems, Series in Modern Condensed Matter Physics, Vol.2, (World Scientific, Singapore, second edition, 1998).

[3] D.M. Eigler and E.K. Schweizer, Nature 344, 524 (1990); A.A. Louis and J.P. Sethna, Phys. Rev. Lett. 74, 1363 (1995).

[4] S. Han, J. Lapointe, and J.E. Lukens, Phys. Rev. Lett. 66, 810 (1991).

[5] M. Sassetti and U. Weiss, Phys. Rev. A 41, 5383 (1990).

[6] M. Sassetti and U. Weiss, Phys. Rev. Lett. 65, 2262 (1990).

[7] S. Chakravarty and J. Rudnick, Phys. Rev. Lett. 75, 501 (1995).

[8] T.A. Costi and C. Kieffer, Phys. Rev. Lett. 76, 1683 (1996); T.A. Costi, Phys. Rev. Lett. 80, 1038 (1998).

[9] K. Völker, cond-mat/9712080 preprint.

[10] R. Egger, H. Grabert, and U. Weiss, Phys. Rev. E 55, R3809 (1997).

[11] F. Lesage and H. Saleur, Phys. Rev. Lett. 80, 4370 (1998).

[12] F. Lesage, H. Saleur, and S. Skorik, Phys. Rev. Lett. 76, 3388 (1996); Nucl. Phys. B 474, 602 (1996).

[13] J.T. Stockburger and C.H. Mak, Phys. Rev. Lett. 80, 2657 (1998).

[14] M. Grifoni, M. Winterstetter, and U. Weiss, Phys. Rev. E 56, 334 (1997).

[15] S. P. Strong, Phys. Rev. E 55, 6636 (1997).

[16] F. Guinea, Phys. Rev. B 32, 4486 (1985).

[17] R. Silbey and A. Harris, J. Chem. Phys. 80, 2615 (1984).

[18] The Hamiltonian $H_{\mathrm{coh}}=-\hbar \Delta \tilde{\sigma}_{x} / 2+H_{\mathrm{R}, \mathrm{I}}$, where $H_{\mathrm{R}, \mathrm{I}}$ is the second term in Eq. (2.1), effectively describes a decoupled system, $\widetilde{H}_{\mathrm{coh}}=U^{-1} H_{\mathrm{coh}} U=-\hbar \Delta \sigma_{x} / 2+H_{\mathrm{R}}$, exhibiting persistent oscillations.

[19] This is equivalent to calculating the autocorrelation function of the bare $\sigma_{x}$ with respect to the transformed Hamiltonian $\widetilde{H}=U^{-1} H U$ [15]. For $K=\frac{1}{2}$, the Hamiltonian $\widetilde{H}$ describes the Toulouse limit of the resonance level model.

[20] H. Grabert, P. Schramm, and G.-L. Ingold, Phys. Rep. 168, 115 (1988).

[21] In order to avoid boundary terms in the influence functional (4.4), we have included a jump at time $t$.

[22] A similar argument was given in Ref. [15] within an imaginary-time approach. 